\title{
The dynamic condylar screw in the management of subtrochanteric fractures: does judicious use of biological fixation enhance overall results?
}

\author{
Manzoor Ahmed Halwai - Shabir Ahmed Dhar • \\ Mohammed Iqbal Wani - Mohammed Farooq Butt · \\ Bashir Ahmed Mir · Murtaza Fazal Ali · Imtiyaz Hussain Dar
}

Received: 2 October 2007/ Accepted: 13 November 2007/Published online: 1 December 2007

(c) Springer-Verlag 2007

\begin{abstract}
Subtrochanteric fractures are fraught with certain anatomic, biologic and biomechanical challenges. Evolution of implants like the Gamma nail, fixed-angle nail plates, compression hip screws and dynamic hip screws with trochanteric stabilization plates underlines a persistent quest for a better implant. We studied the dynamic condylar screw DCS as an implant on a series of 30 consecutive patients with subtrochanteric fractures. Our purpose was to assess this implant as a panacea for subtrochanteric fractures. All cases of AO type A and B were anatomically fixed, whereas type $\mathrm{C}$ was biologically plated. The idea was to assess the applicability and adaptability of the DCS. Fractures in 29 cases united, with one patient suffering from an implant failure. There were 17 excellent, 5 good, 5 fair and 3 poor results. The DCS is a definite advance over previous methods of treatment; when combined with the utilization of biological fixation techniques for comminuted fractures, can be relied upon to treat all types of subtrochanteric fractures.
\end{abstract}

Keywords The dynamic condylar screw . Subtrochanteric fracture $\cdot$ Biological fixation

The DCS is a satisfactory implant in technologically less advanced settings and can be used as the treatment modality of choice in subtrochanteric fractures in such settings.

M. A. Halwai · S. A. Dhar $(\square) \cdot$ M. I. Wani ·

M. F. Butt - B. A. Mir · M. F. Ali · I. H. Dar

The Government Bone and Joint Surgery Hospital,

Barzallah Srinagar, Kashmir 190005, India

e-mail: shabirdhar@yahoo.co.in

\section{Introduction}

Subtrochanteric fractures of the femur account for 10-34\% of all hip fractures [1]. These fractures are known to be difficult to treat successfully [2]. Certain anatomic, biologic and biomechanical features make this area a unique proposition for the treating surgeon. The subtrochanteric region of the femur is mainly cortical due to which the area of healing as well as the vascularity is poorer, prolonging the healing time. The forces in this area are up to 1,200 pounds/square inch on the medial cortex leading to immense stresses in the area. Besides this the orientation of muscle forces in this area causes shear at the fracture site [3]. During the past 30 years, there has been a near-complete elimination of nonoperative treatment in adults and a corresponding increase in the operative treatment of subtrochanteric fractures [4].

No single implant is universally recommended for the internal fixation of these fractures, and hence periodically new fixation devices are introduced [1]. More recently, the importance of maintaining the blood supply and vitality of all fragments by avoidance of medial dissection has been emphasized [3,5]. This study was based on the results of a prospective study conducted by one unit of our hospital on a consecutive group of 30 patients presenting with subtrochanteric fractures to the casualty department. All 30 fractures were fixed with the DCS implant. The idea of the study was to assess this fixation method in all types of subtrochanteric fractures.

\section{Materials and methods}

This study was conducted prospectively on a group of 30 patients with subtrochanteric fractures who reported to our 
hospital from January 2002 to December 2004. During this period 34 cases with subtrochanteric fracture presented to the unit conducting the study. Four cases were excluded; one open fracture, two pathological fractures and another with an ipsilateral open tibial fracture. Two surgeons conducted all operative procedures.

All fractures were classified radiologically in consultation with a radiologist on the basis of the classification proposed by the AO group, i.e., Muller et al. [6].

Preoperatively, all patients were placed on skeletal traction. Chest physiotherapy and quadriceps exercises were demonstrated to all patients. The patients were placed on DVT prophylaxis with Heparin 5,000 IU subcutaneously twice a day. Preoperative planning included radiographs of the opposite femur to determine the DCS entry site and the proper length of the plate.

\section{Surgical technique}

With the patient lying supine on a traction table, a posterolateral approach was made to the subtrochanteric region. A guide wire was placed on the anterior surface of the femoral neck to determine the anteversion. Another guide pin was inserted across the femoral neck, with due consideration paid to the degree of anteversion and the fixed angle of the implant, such that the tip of the guide wire was seated $2 \mathrm{~cm}$ short of the articular surface in the lower half of the femoral head. An appropriate length condylar screw was then applied over the guide. The condylar screw was engaged in the subchondral bone of the lower quadrant of the femoral head. A suitable side plate was applied after attempting anatomic reduction. At least four screws were applied distal to the main fracture. Minor fragments were lagged in an attempt to restore medial bone support. Bone grafting was carried out in cases of severe posteromedial comminution in Type A and $\mathrm{B}$ fractures where it was not possible to hold the posteromedial area with screws in spite of the anatomical alignment (Figs. 1, 2).

In biologically plated fractures reduction was done using the fracture table utilizing skeletal traction. The focus was on obtaining the length, mechanical and rotational alignments. Correct alignment and rotation were checked intraoperatively using $\mathrm{X}$-ray guidance. The length, axial alignment and rotation, were checked again using clinical assessment. Two separate incisions were made. The DCS screw was placed as mentioned from the proximal incision. From the proximal incision the plate was slid across the fracture extraperiosteally with the barrel facing laterally. Once the plate reached the proper length it was rotated and the barrel slid over the condylar screw. The length of plate chosen was such that the four holes of the plate extended beyond the distalmost extent of the fracture. The plate in this area was exposed by a second incision and fixed to the bone by cortical screws. During the whole procedure the fracture was not exposed. The incision was closed over a suction drain.

Postoperatively, quadriceps exercises were encouraged on the first postoperative day. Range of motion exercises were started within the limits of pain. Partial weight bearing was commenced in type A and B fractures from the second week onwards as dictated by the patient's tolerance to pain. Postoperatively, the patients were put on Cefazolin $500 \mathrm{mg} \mathrm{I} / \mathrm{V}$ eight hourly, for 5 days. The patients were discharged on the sixth day post operatively. Stitch removal was done on the 14th day. In patients with type $\mathrm{C}$ fractures weight bearing was not commenced until 6 weeks.

Regular check ups were made at 4, 8, 12, and 24 weeks. The final assessment was done at 1 year on the basis of the criteria laid down by Radford et al. [7] (Table 1).
Fig. 1 Anatomical reduction and fixation
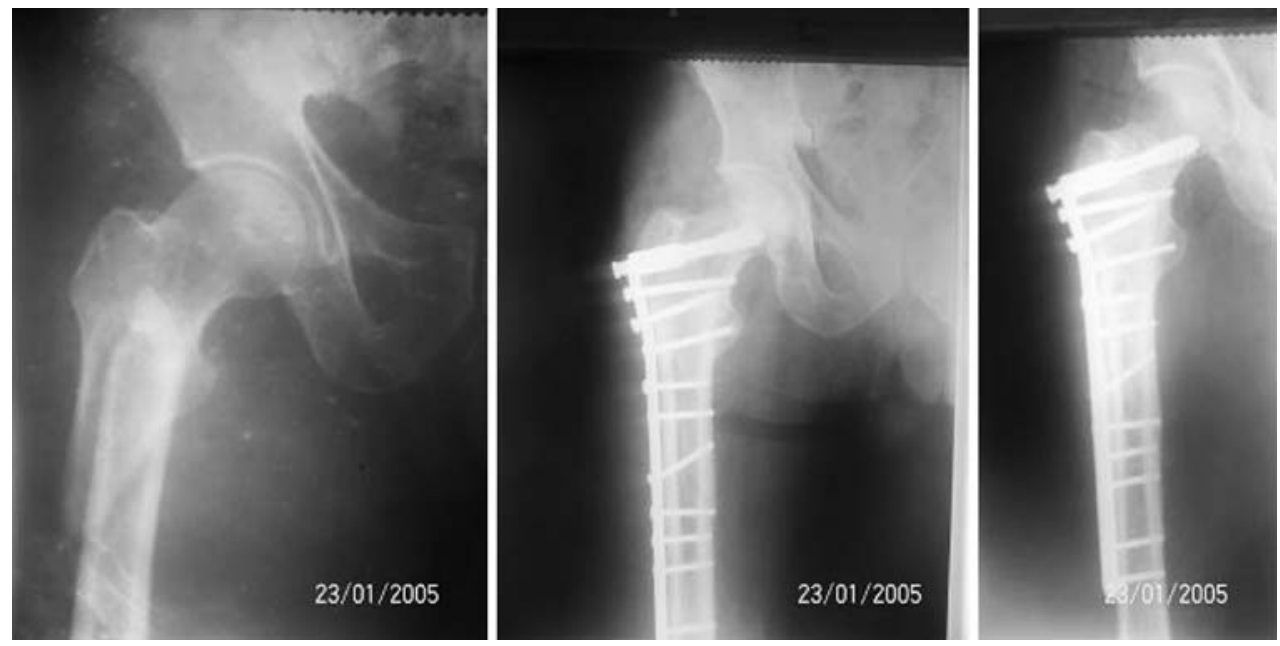
Fig. 2 Biological fixation and union


Table 1 Criteria for the assessment of results

\begin{tabular}{ll}
\hline Excellent & Flexion loss of less than 10 degrees \\
& No varus, valgus or rotatory deformity \\
& No pain \\
& Perfect joint congruity \\
& Not more than one of the following \\
Good & Loss of length not more than $1-2 \mathrm{~cm}$ \\
& Less than 10 degrees varus or valgus deformity \\
& Flexion loss not more than 20 degrees \\
& Minimal pain \\
Fair & Any of the two criteria in the good category \\
Failure & Flexion less than 90 degrees \\
& Varus or valgus exceeding 15 degrees \\
& Joint incongruency \\
& Disabling pain \\
\hline
\end{tabular}

\section{Results}

The age range of the patients in our study was 22-68 years with a mean age of 52.7 years. Six [20\%] patients were in the age group of $22-40$ years. Eighteen patients $\{60 \%\}$ were in the age group of 41-60 years and six patients [20\%] from 61 to 70 years. Males comprised 23\{77\%\} of our cases and females $7\{23 \%\}$. The right limb was involved in $19\{64 \%\}$ patients and the left limb in $11\{36 \%\}$ of our cases. Eighteen patients were injured in a road traffic accident and 12 patients in a fall from height. Eighty-three percent, i.e., 25 patients reported to the hospital within $24 \mathrm{~h}$ and the rest within 1 week of sustaining the trauma. The time interval between injury and admission averaged 1.4 days.

According to the $\mathrm{AO}$ classification 15, i.e., $50 \%$ of the fractures were type A, 8 fractures, i.e., $24 \%$ were type B, and 7 , i.e., $26 \%$ were of type C. Average time interval between admission and surgery was 11.4 days with a range of 6-20 days. Twenty-one patients were operated under spinal anesthesia and 9 patients under general anesthesia. In our series of 30 patients, primary bone grafting was done
Table 2 Complications

\begin{tabular}{llll}
\hline $\begin{array}{l}\text { Serial } \\
\text { number }\end{array}$ & Complication & $\begin{array}{l}\text { Number } \\
\text { of cases }\end{array}$ & Percentage \\
\hline 1. & Wound infection & 02 & 07 \\
2. & Limb length discrepancy & 05 & 17 \\
3. & Pain on walking & 02 & 07 \\
4. & Restricted knee movement & 06 & 20 \\
5. & Restricted hip movement & 02 & 07 \\
5. & Loosening of implant & 01 & 03 \\
6. & Deformity & 08 & 27 \\
7. & Foot drop & 01 & 03 \\
8. & Implant breakage & None & 00 \\
9. & Complications of & None & 00 \\
10. & recumbency & & \\
\hline
\end{tabular}

in only 3 patients. These cases were elderly patients with significant posteromedial comminution (more than $1 / 3$ diameter), which even though reduced was not held with screws. All three were type B fractures. In these cases no complication of bone grafting occurred. The average duration of surgery was 80 min with only two cases taking longer than $120 \mathrm{~min}$. One patient had loosening of the implant and required exchange and bone grafting. The fracture united by 48 weeks. The average time for union was 19.7 weeks. In two cases in which bone grafting was done, the bones united by 20 weeks. The complications encountered in our series are summarized in Table 2. The foot drop that occurred in one case, which was possibly due to traction injury, resolved in 8 weeks. In the meantime, the patient ambulated on an orthosis.

Of the eight cases with deformity four (13\%) had varus and two were having a greater than 10 degrees varus. Two patients had a valgus of 10 degrees, whereas two patients had an external rotation deformity of 10 degrees. Union in these cases occurred uneventfully. Four of our patients had a shortening of $2 \mathrm{~cm}$ and one had a shortening of $1 \mathrm{~cm}$. 
One patient complained of pain at rest on final follow-up and two patients complained of pain on full weight bearing. The range of motion achieved in our patients is shown in Table 2.

Evaluation on the basis of the Radford [7] criteria showed 17 excellent, 5 good, 5 fair and 3 poor results. At the time of final follow up all the patients were ambulatory including the patient who required a revision surgery due to implant failure.

\section{Discussion}

Subtrochanteric fractures of the femur demand a special consideration in orthopedic traumatology, given the high rate of complications associated with their management [8]. Intramedullary devices have an advantage of reducing the moment arm over which bending forces act compared with a laterally placed plate [9]. However, in cases where the medial femoral cortex is comminuted, the use of conventional femoral interlocking nails makes for an unstable biomechanical construct [10]. Several other implants have evolved over time to manage this difficult fracture. These include nails, blade plates and Kuntscher rods as reported by Seinsheimer [11]. Velasco [12] reported the use of the Massie nail and the Jewett nail. The sliding screw is technically straightforward to use, but because of its 135-degree screw plate angle, it may not be possible to supplement the sliding screw with additional cortical screws in the proximal fragment of a subtrochanteric fracture [13].

When the intramedullary devices cannot be used for technical reasons, the dynamic condylar screw provides a reasonable alternative, especially in the third world where costly image intensifier facilities are not available as a norm in hospitals due to financial constraints. Because of the familiarity of most surgeons with the sliding hip screw techniques in the treatment of trochanteric fractures, the adaptation is enhanced. The position of the condylar screw makes it possible to stabilize the proximal fragment and hence the fracture with extra screws. Nungu et al. [14] felt that the DCS is able to tolerate bending loads well. They however recommended the reconstruction of a good medial support.

Our study was based on the recommendations of Nungu et al. [14], Radford et al. [7], and Schatzker [15]. In cases with increasing comminution it is worthwhile incorporating the principles of biological fixation. These indirect reduction techniques were initially reported with the condylar blade plate. Due to the three-dimensional exactness required, the technique proved to be difficult. The DCS facilitates rotation and allows sagittal plane adjustment [5, 16]. The complete restitution of medial cortical support may not be achievable with indirect reduction techniques; even so, the preservation of the vascularity of small fragments appears to offset this biomechanical disadvantage [17].

In most of the series where DCS fixation has been used, either open reduction or biologic fixation has been studied separately [7, 14, 18, 19]. Our experience suggests that it is not possible to apply biological reduction in all cases as alignment requires open reduction. Conversely, it is not easy to accurately reduce type $\mathrm{C} 1$ and $\mathrm{C} 2$ fractures. Through using biological techniques for comminuted type C fractures or for those types A and B patterns, which reduce well simply on traction, and leaving open reduction only for those fractures, which need reconstruction of the medial buttress we were able to obtain a union rate of $96.6 \%$. The lone case where implant loosening occurred could be put down to technical faults. In attempting to reconstruct the medial buttress the drill bit broke and a proper lag effect was not achieved. Additionally, the number of screws distal to the fracture was insufficient. Our surgical time averaged $80 \mathrm{~min}$, which was less than the series of Vaidya et al. [19]. The results of our series in comparison to other series in terms of implant failure is shown in Table 3. Pakuts [20] had one implant failure and one varus malunion in 15 cases. Comparison of implant failure with other studies shows that Asher et al. [2[t11]] had a failure rate of $33 \%$ with Blade plates, and Ruff et al. [21] a failure rate of $11 \%$ with DHS.

Our study even though conducted on a relatively small number of cases avoids selection bias in that almost all cases of subtrochanteric fracture were included.

\section{Conclusion}

The dynamic condylar screw is a safe and reliable implant for the management of subtrochanteric fractures with predictable results when principles of open reduction and internal fixation, biological reduction and bone grafting are followed as indicated. It can be recommended as an implant of choice in settings where image intensifier facilities are limited and costs of modern cephalomedullary nails prohibitive to many patients.

Table 3 Implant failure rates of our series as compared with other series

\begin{tabular}{ll}
\hline Radford et al. [7] & (11) $1 / 11,9 \%$ \\
Warwick et al. [13] & (20) $6 / 36,17 \%$ \\
Nungu et al. [14] & (9) $3 / 15,20 \%$ \\
Schatzker et al. [15] & (13) $2 / 6,33 \%$ \\
Vaidya et al. [19] & (17) $0 / 31,0 \%$ \\
This series & $1 / 30,3.33 \%$ \\
\hline
\end{tabular}




\section{References}

1. Lavelle David G (2003) Campbells operative orthopaedics, 10th edn. Mosby, St. Louis, p 2897

2. Asher MA, Tippet JW, Rockwood CA, Zilber S (1976) Compression fixation of subtrochanteric fractures. CORR No 117: June

3. Sims SH (2002) Treatment of complex fractures. Orthop Clin North Am 33(1):1-12

4. Wadell JP (1979) Subtrochanteric fractures of femur: a review of 130 patients. J Trauma 19(8):582-92

5. Kinast C, Bolhofner BR, Mast JW, Ganz R (1989) Subtrochanteric fractures of the femur. Results of treatment with the 950 condylar blade plate. Clin Orthop 238:122

6. Muller ME, Nazarian S, Koch P et al (1990) The AO classification of fractures of long bones. Springer, Berlin, p 116

7. Radford PJ, Howell CJ (1992) The AO dynamic condylar screw for fractures of the femur. Injury 23:89-93

8. Bedi A, Le TT (2004) Subtrochanteric femur fractures. Orthop Clin North Am 35:473-483

9. Tencer AF, Johnson KD, Johnston DW et al (1984) A biomechanical comparison of various methods of stabilization of subtrochanteric fractures of femur. J Orthop Res 2:297-305

10. Fielding JW, Magliato HJ (1966) Subtrochanteric fractures. Surg Gynaecol Obstet 122:297-305

11. Seinsheimer F (1978) Subtrochanteric fractures of the femur. JBJS 60-A(3):300-306
12. Velasco UR, Comfort TH (1978) Analysis of treatment problems in subtrochanteric fractures of the femur. J Trauma 18(7):513523

13. Warwick DJ, Crichlow TPKR, Langkamer VG et al (1995) The dynamic condylar screw in the management of subtrochanteric fractures of the femur. Injury 26(4):241-244

14. Nungu KS, Olerud C, Rehnberg L (1993) Treatment of subtrochanteric fractures with the $\mathrm{AO}$ dynamic condylar screw. Injury 24(2):90-2

15. Schatzker J, Mahomed N, Schiffman K, Kellam J (1989) Dynamic condylar screw: a new device. J Orthop Trauma 3:124

16. Blatter G, Janssen M (1994) Treatment of subtrochanteric fractures of the femur: reduction on the traction table and fixation with dynamic condylar screw. Arch Orthop Trauma Surg 113(3):138-41

17. Perren SM (1989) Trends in the internal fixation. Potential, limits and requirements. Injury 30(suppl 2):SB2-SB4 [Editorial]

18. Kulkarni SS, Moran CG (2003) Results of dynamic condylar screw for subtrochanteric fractures. Injury 34:117-122

19. Vaidya SV, Dholakia DB, Chaterjee A (2003) The use of a dynamic condylar screw and biological reduction techniques for subtrochanteric femur fracture. Injury 34:123

20. Pakuts AJ (2004) Unstable subtrochanteric fractures gamma nail versus dynamic condylar screw. Int Orthop 28(1):21-4. Epub 2003 Aug 26

21. Ruff ME, Lubbers LM (1986) Treatment of subtrochanteric fractures with a sliding plate device. J Trauma 26:75 